\title{
Un cariotipo del murciélago sedoso de cola corta (Carollia brevicauda [Schinz, 1821], Chiroptera: Phyllostomidae) de los andes de Colombia
}

\author{
Elkin A. Noguera-Urbano ${ }^{1,2^{*}}$ y Silvia Muñoz-Montenegro ${ }^{2}$
}

Introduction: The only record of a Carollia brevicauda (Schinz, 1821) karyotype in Colombia to date was described from a single specimen collected from Leticia (department of Amazonas, Colombia). In this study, we offer a description of the karyotype of a female specimen of $C$. brevicauda, collected at 1,647 $\mathrm{m}$ in the locality of Chachagüi, in the Colombian Andes.

Methodology: The karyotype was obtained from bone marrow and conventional staining with Giemsa. We describe the chromosomal number, fundamental number and the karyotypic pattern. We compared the karyotype with others proposed for the species and several closely related species.

Results: This study increased the number of karyotypes reported for Colombian populations of $C$. brevicauda. The karyotype of $C$. brevicauda described in this paper agrees with karyotypes of other populations with $2 \mathrm{n}=20$ and $\mathrm{FN}=36$. The karyotypic pattern includes one sexual pair, two subtelocentrics pairs, two submetacentrics pairs and five metacentrics pairs.

Discussion: The pattern recorded herein is different to other reported for the species and the genus. The descriptions of variations in chromosomal morphology in C. brevicauda are incomplete, but it is possible that the different karyotypic patterns are indicators of polymorphism within the genus. Other studies are necessary to describe the yet undescribed karyotypes of C. manu and C. monohernandezi and to obtain additional evidence for the phylogenetic reconstructions of Carollia.

Key words: bat; chromosomes; genetics; genotype; mammal; variation.

\section{Resumen}

Se reporta el cariotipo de un ejemplar hembra C. brevicauda capturado en el municipio de Chachagüi, Colombia. El cariotipo fue obtenido a partir de médula ósea con tinción convencional de Giemsa. Con este reporte se incrementó a dos el número de reportes citogenéticos de C. brevicauda para poblaciones de Colombia. El cariotipo de C. brevicauda analizado presentó $2 \mathrm{n}=20$ y NF =36, mientras que la fórmula cromosómica del ejemplar esta compuesta por un par sexual, dos pares subtelocéntricos, dos pares submetacéntricos y cinco pares metacéntricos. Se determinaron variaciones en la morfología de los cromosomas, pero uniformidad en el número cromosómico. La información disponible indica la necesidad de estudios para describir los cariotipos de C. manu y C. monohernandezi.

\footnotetext{
${ }^{1}$ Museo de Zoología "Alfonso L. Herrera", Departamento de Biología Evolutiva, Facultad de Ciencias, Universidad Nacional Autónoma de México, Coyoacan 04510 México. E-mail: elkalexno@gmail.com (EAN-U)

${ }^{2}$ Asociación GAICA. Pasto, Nariño, Colombia. E-mail: silvimomu@gmail.com (SM-M)

*Corresponding author
} 
Palabras clave: cromosomas; genética; genotipo; mamífero; murciélago; variación.

Los murciélagos pertenecientes al género Carollia Gray, 1838 se distribuyen desde México hasta Paraguay (McLellan y Koopman 2008), en un rango altitudinal entre 0 y 2,660 m (Muñoz 1995). En Colombia se han reportado cinco especies de Carollia, de las cuales cuatro son aceptadas actualmente (C. castanea H. Allen 1890, C. brevicauda, C. perspicillata [Linnaeus 1758] y C. monohernandezi Muñoz et al. 2004) y una se encuentra aún sin describir (Solari et al. 2013). Tres especies se distribuyen en el suroccidente del país: C. castanea, C. perspicillata y C. brevicauda (Solari et al. 2013). En particular, poblaciones de C. brevicauda se han reportado a lo largo de los Andes centrales y orientales de Nariño, en los municipios de Ancúya, Barbacoas, Chachagüi, Pasto, Ricaurte y San Lorenzo (Ramírez-Chaves y Noguera-Urbano 2010).

Las poblaciones de C. brevicauda muestran una amplia variabilidad morfológica atribuida a plasticidad por presiones ambientales (York y Papes 2007) y han sido objeto de estudios genéticos y filogenéticos como complejo de especies (Baker et al. 2002).

Otro tipo de información se ha reportado con el fin de explicar dicha variabilidad, desde la descripción de patrones biogeográficos (Hoffman y Baker 2003), caracterizaciones morfológicas (Pine 1972; McLellan 1984; Muñoz 1995; McLellan y Koopman 2008), cromosómicas (Patton y Gardner 1971; Stock 1975; Baker y Bleier 1971; Parish et al. 2003; Pieczarka et al. 2005; Noronha et al. 2009) y/o moleculares de algunas de sus poblaciones (Baker et al. 2002; Hoffman y Baker 2003; Solari y Baker 2006); pese a ello se desconoce información sobre los cromosomas de la especie en poblaciones andinas de Colombia.

En estudios previos se ha reportado que el cariotipo de C. brevicauda presenta un número cromosómico 2n = 20 hembras - 21 machos (Patton y Gardner 1971; Stock 1975; Parish et al. 2003; Pieczarka 2005). De manera general, para C. brevicauda se ha descrito una formula cariotípica compuesta por cuatro pares metacéntricos (4M), dos pares submetacéntricos (2SM) y tres pares subtelocéntricos (3ST); el número fundamental (NF) es 36. En las hembras el par sexual $X$ es subtelocéntrico (ST), mientras que en los machos el cromosoma $X$ es subtelocéntrico (ST) y los cromosomas $Y_{1}$ y $Y_{2}$ son dos pequeños acrocéntricos (Stock 1975). Algunos reportes sobre el cariotipo de $C$. brevicauda provienen del Perú y Brasil (Patton y Gardner 1971; Stock 1975; Pieczarka 2005). En Colombia el único reporte del cariotipo de C. brevicauda podría ser el de Baker y Bleier (1971) a partir de un ejemplar capturado en Leticia en el departamento de Amazonas (ejemplar Z137) identificado inicialmente como C. subrufa ( $2 n=20$ - 21).

Las características cromosómicas de Carollia son indicadoras de la diversidad genética del género y pueden ser importantes herramientas para el estudio de especies crípticas dentro del grupo (Stock 1975; Baker et al. 2002; Solari y Baker 2006), por lo tanto en este estudio se presenta el cariotipo de un ejemplar hembra de C. brevicauda proveniente de los Andes Colombianos.

El ejemplar fue capturado en el municipio de Chachagüí $\left(1.42^{\circ} \mathrm{N}-77.27^{\circ} \mathrm{W}, 1,647\right.$ m), departamento de Nariño, Colombia. La localidad de muestreo se caracteriza por la presencia de parches de bosque y un avanzado grado de fragmentación, el ecosistema 


\section{Material}

es del tipo sub-xerofítico (obs. pers.). La piel y el cráneo del ejemplar analizado (MUN596 [EK058]) se encuentran depositados en el Museo de Historia Natural Universidad de Nariño (MUN), Pasto, Colombia. El ejemplar fue identificado considerando caracteres morfológicos y morfométricos de la especie (McLellan y Koopman 2008) y con la revisión de material de referencia en los museos de historia natural de las universidades de Nariño (MUN), del Valle (UV) y Antioquia (CTUA).

\section{y $\underline{\text { Métodos }}$}

La preparación cromosómica fue realizada a partir de medula ósea de húmero y tinción estándar con Giemsa (Baker et al. 2003). Los cromosomas se clasificaron considerando la relación entre brazo largo y corto (RB; Levan et al. 1964); además se determinó el número diploide (2n) con el conteo de cromosomas en 10 células metafásicas y el número fundamental (NF) identificado como el número total de los brazos de los autosomas. El cariotipo fue organizado de acuerdo a Baker y Bleier (1971). Para comparar la variación de los cariotipos en poblaciones de la especie y otras congéneres, se revisó literatura referente a las especies del género.

El ejemplar presentó $2 \mathrm{n}=20$ (Fig. 1 A y B) y NF = 36. La fórmula cromosómica estuvo compuesta por dos pares de cromosomas subtelocéntricos (RB: 3.5, 3.1), dos pares submetacéntricos (RB: 2.1, 1.8) y cinco pares metacéntricos (RB: 1.4, 1.4, 1.3, 1.3, 1.3).

A

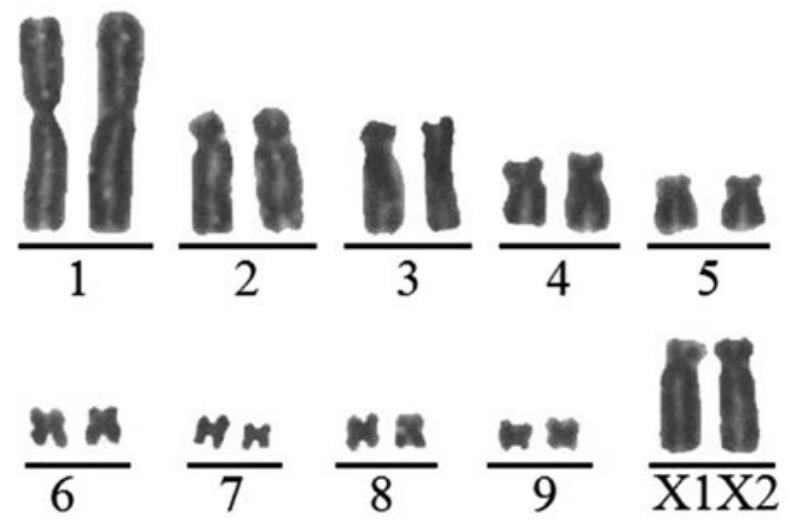

Figura 1. Cromosomas del ejemplar hembra de Carollia brevicauda (MUN-596). (A) Cariotipo e idiograma de $C$. brevicauda y (B) Cariotipo extendido en metafase de médula ósea.

\section{B}

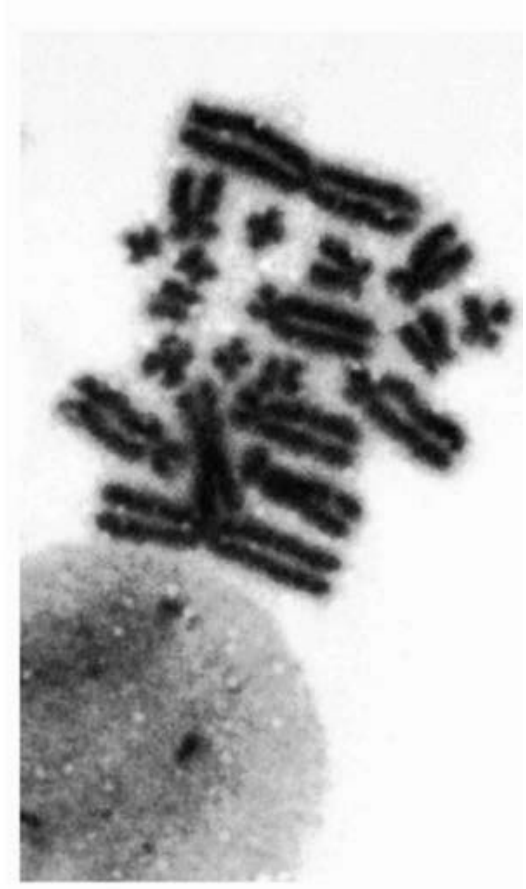

La formula obtenida fue X1X2:2:2:5 (Grupo 1: Sexual ST, Grupo 2: Par 3 y 4 ST, Grupo 3: Par 5 y 6. SM, Grupo 4: Par 2, 7, 8, 9, 10. M). El cariotipo del ejemplar estudiado no presentó diferencias en cuanto a número de cromosomas y número fundamental, sin embargo la formula cromosómica es distinta a la reportada para la especie (Tabla 1). 
Hasta este reporte, el cariotipo de C. brevicauda en Colombia era incierto, ya que Baker y Bleier (1971) describieron el cariotipo de un ejemplar de C. subrufa de Leticia, departamento de Amazonas, ejemplar que en la actualidad ha sido catalogado como $C$. brevicauda (TTU 8837 [TK 137]). Considerando que C. subrufa no tiene distribución conocida para Colombia (Solari et al. 2013), el reporte de Baker y Bleier (1971) sería el primer reporte del cariotipo de $C$. brevicauda en el país y el reportado en este estudio (Fig. 1) posiblemente es el segundo. Los resultados demuestran que el ejemplar de C. brevicauda presenta características típicas de la especie en cuanto al número cromosómico y número fundamental (Patton y Gardner 1971; Stock 1975; Baker y Bleier 1971; Parish et al. 2003; Pieczarka et al. 2005; Noronha et al. 2009). Asumiendo que la población C. brevicauda analizada presenta dimorfismo en los cromosomas sexuales como es frecuente en la especie y otras especies del género (Baker 1967; Hsu et al. 1968; Baker y Hsu 1970; Baker y Bleier 1971; Patton y Gardner 1971; Cloutier y Thomas 1992; Santos y de Souza 1998; Noronha et al. 2009), probablemente los machos de la población muestreada presentaran $2 n=21$. Sin embargo es necesario describir un mayor número de ejemplares e incluir a machos.

Para los murciélagos del género Carollia la homogeneidad en el número cromosómico ya ha sido documentada (Patton y Gardner 1971; Stock 1975; Pieczarka et al. 2005), se sugiere que los números cromosómico y fundamental en Carollia son estables y se han logrado mantener así aproximadamente desde hace siete millones de años (Parish 2003), con excepción de C. benkeithi (Tabla 1; Patton y Gardner 1971; Solari y Baker 2006).

El cariotipo del ejemplar de C. brevicauda analizado presento un par sexual similar al de otras hembras de la especie (Patton y Gardner 1971) y otras especies del género (Baker 1967; Hsu et al. 1968; Baker y Hsu 1970; Baker y Bleier 1971; Stock 1975; Santos y de Souza 1998; Baker et al. 2002; Parish et al. 2003; Pieczarka 2005; Solari y Baker 2006). La fórmula de los autosomas descrita en este estudio es diferente a otras mencionadas en la literatura para la especie (Baker y Bleier 1971; Stock 1975), pero comparte con la reportada por Stock (1975) la presencia de dos pares submetacéntricos.

Los cambios en la fórmula cariotípica de los cromosomas han sido reportados para otras especies de Carollia (Baker 1967; Patton y Garder 1971; Stock 1975), pero no se ha sugerido ningún tipo de relación con ordenamientos morfológicos o genéticos que son evidentes en análisis filogenéticos del género (Baker y Bleier 1971; Patton y Garder 1971; Hoffman y Baker 2003; Solari y Baker 2006).

Resultados

Con el presente trabajo se incrementa la representatividad geográfica de los estudios citogenéticos de C. brevicauda de los Andes. En Colombia, el conocimiento sobre las características cromosómicas de las poblaciones de murciélagos de Carollia y otros géneros es precario, por lo cual es necesario continuar con este tipo de estudios, ya que se reconoce que la citogenética aporta en los análisis de especies crípticas (Baker et al. 2002; Solari y Baker 2006) y permite proponer explicaciones sobre la evolución de las especies. Para C. manu del Perú y C. monohernandezi de Colombia se desconocen los cariotipos, mientras que de C. benkeithi y C. sowelli la información aun es escasa, por lo tanto sugerimos que estudios citogenéticos adicionales se podrían enfocar en las cuatro especies. 


\begin{tabular}{|c|c|c|c|c|c|c|c|c|}
\hline \multirow{3}{*}{$\begin{array}{l}\text { Tabla 1. Variación } \\
\text { cromosómica publicada } \\
\text { para el género Carollia. } \\
\text { En cariotipo las letras } \\
\text { indican la morfología } \\
\text { de los cromosomas }\end{array}$} & \multirow[b]{2}{*}{ Especie } & \multicolumn{6}{|c|}{ Cariotipo } & \multirow[b]{2}{*}{ Fuente y Observaciones } \\
\hline & & $2 n$ & NF & Autosomas & $X$ & $\mathrm{Y}_{1}$ & $\mathrm{Y}_{2}$ & \\
\hline & C. brevicauda & 20 & 36 & $2 \mathrm{ST}+2 \mathrm{SM}+5 \mathrm{M}$ & ST & & & Este estudio. \\
\hline $\begin{array}{l}\text { (A: acrocéntricos; M: } \\
\text { metacéntricos; } \quad \text { SM: } \\
\text { submetacéntricos y ST: } \\
\text { subtelocéntrico). }\end{array}$ & C. brevicauda & $20-21$ & 36 & $\begin{array}{l}3 \mathrm{ST}+2 \mathrm{SM}+4 \mathrm{M} \\
2 \mathrm{ST}+1 \mathrm{SM}+6 \mathrm{SM}\end{array}$ & $\begin{array}{l}\text { ST } \\
\text { ST }\end{array}$ & $\begin{array}{l}\text { A } \\
A\end{array}$ & $\begin{array}{l}\text { A } \\
A\end{array}$ & $\begin{array}{l}\text { Patton y Gardner 1971; Stock } 1975 \\
\text { (formula); Baker y Bleier } 1971 \\
\text { (ejemplar Colombia); Parish et al. } \\
\text { 2003, Pieczarka et al. } 2005 \text { (CS-B); } \\
\text { Noronha et al. } 2009 \text { (CS-B) }\end{array}$ \\
\hline & C. perspicillata & $20-21$ & 36 & $\begin{array}{l}3 \mathrm{ST}+4 \mathrm{M}+2 \mathrm{SM} \\
2 \mathrm{ST}+1 \mathrm{SM}+6 \mathrm{SM} \\
4 \mathrm{M}+2 \mathrm{SM}+3 \mathrm{SA}\end{array}$ & $\begin{array}{l}\text { ST } \\
\text { ST } \\
\text { SA }\end{array}$ & $\begin{array}{l}A \\
A \\
A\end{array}$ & $\begin{array}{l}\text { A } \\
A \\
A\end{array}$ & $\begin{array}{l}\text { Baker } 1967 \text { (formula); Hsu et al. } \\
\text { 1968, Baker y Hsu } 1970 \text { (NC-CS); } \\
\text { Baker y Bleier } 1971 \text { (ejemplar } \\
\text { Colombia); Patton y Gardner 1971; } \\
\text { Cloutier y Thomas } 1992 \text { (formula); } \\
\text { Santos y de Souza } 1998 \text { (CS y } \\
\text { bandas); Noronha et al. } 2009 \text { (CS-B) }\end{array}$ \\
\hline & C. subrufa & $20-21$ & 36 & $3 S T+4 M+2 S M$ & ST & A & A & $\begin{array}{l}\text { Baker } 1967 \text { (formula); Hsu et. al. } \\
1968 \text { (CS) }\end{array}$ \\
\hline & C. castanea & 20 & & $\begin{array}{l}3 S T+4 M+2 S M \\
2 S T+1 S M+6 S M\end{array}$ & $\begin{array}{l}\text { ST } \\
\text { ST }\end{array}$ & A & A & $\begin{array}{l}\text { Stock } 1975 \text { (formula); Baker y } \\
\text { Bleier } 1971 \text { (ejemplar Colombia) }\end{array}$ \\
\hline (?) El símbolo de & C. benkeithi & 22 & & $2 \mathrm{ST}+1 \mathrm{~A}+6 \mathrm{SM}$ & SM & A & & $\begin{array}{l}\text { Patton y Gardner } 1971 \text { (formula); } \\
\text { Solari y Baker } 2006 \text { (CS) }\end{array}$ \\
\hline $\begin{array}{l}\text { interrogación indica la } \\
\text { ausencia del dato para }\end{array}$ & C. manu & $?$ & & & & & & No conocido \\
\hline $\begin{array}{l}\text { la especie, NC: número } \\
\text { cromosómico, CS: } \\
\text { cromosomas sexuales y }\end{array}$ & C. monohernandezi & $?$ & & & & & & No conocido \\
\hline B: bandas. & C. sowelli & 21 & 36 & $3 \mathrm{ST}+4 \mathrm{M}+2 \mathrm{SM}$ & ST & A & & $\begin{array}{l}\text { Baker et al. } 2002 \text { (Cariotipo similar } \\
\text { al de C. brevicauda) }\end{array}$ \\
\hline
\end{tabular}

\section{Agradecimientos}

\section{Literatura citada}

A S. Solari de la Colección Teriológica de la Universidad de Antioquia (CTUA) y O. Murillo de la Universidad del Valle (UV) por permitir el acceso a los ejemplares bajo su cuidado. A S. Solari por los comentarios sobre ejemplares de Carollia de la Amazonia y a D. Zurc por la colaboración en la revisión de ejemplares. A D. Tirira, H. RamírezChaves, J. J. Calderón y R. Prieto por los comentarios realizados a una versión inicial del documento. A la Asociación GAICA por los equipos y a la VIPRI de la Universidad de Nariño por la beca para el proyecto de grado "Cariología comparada de C. perspicillata y C. brevicauda en Nariño- Colombia", realizado por el primer autor. EANU agradece al posgrado en Ciencias Biológicas de la Universidad Nacional Autónoma de México (UNAM) y al programa de becas de posgrado de CONACyT por el soporte dado durante las adecuaciones finales de este documento. A J. E. Maldonado y dos revisores anónimos que aportaron con comentarios y sugerencias para el mejoramiento del documento.

Baker, R. J., S. Solari, y F. G. Hoffmann. 2002. A new Central American species from the Carollia brevicauda complex. Occasional Papers of the Museum of Texas Tech University 217:1-16.

BAKER, R. J. 1967. Karyotypes of bats of the family Phyllostomidae and their taxonomic 
implications. Southwestern Naturalist 12:407-428.

BaKer, R. J., Y T. C. Hsu. 1970. Further studies in the sex chromosome systems of American leaf-nosed bats (Chiroptera: Phyllostomatidae). Cytogenetics 9:131-138.

Baker, R. J., M. Hamilton, y D. A. Parish. 2003. Preparations of mammalian karyotype under field conditions. Occasional Papers, Museum of Texas Tech University 228:1-8.

BAKER, R. J., Y W. J. Bleier. 1971. Karyotypes of bats of the subfamily Carollinae (Mammalia: Phyllostomatidae) and their evolutionary implications. Experientia 27:220-222.

Hoffman, F. G., y R. J. BaKer. 2003. Comparative phylogeography of short-tailed bats (Carollia: Phyllostomidae). Molecular Ecology 12:3403-3414.

Hsu T., R. J. Baker, y T. UtakoJ.. 1968. The multiple sex chromosome system of American leafnosed bats (Chiroptera, Phyllostomatidae). Cytogenetics 7:27-38.

Levan, A., K. Fredga, y A. A. Sandberg. 1964. Nomenclature for centromeric position on chromosomes. Hereditas 52:201-220.

McLellan, L. A. 1984. Morphometric analysis of Carollia (Chiroptera, Phyllostomidae). American Museum Novitates 2791:1-35.

McLellan, L. J., y K. F. Koopman. 2008. Subfamily Carolliinae. Pp. 211 en Mammals of South America: marsupials, xenarthrans, shrews and bats (Gardner, A. L., ed.). The University of Chicago Press. Chicago, EE.UU.

Muñoz, J. 1995. Clave de murciélagos vivientes en Colombia. Editorial Universidad de Antioquia. Medellín, Colombia.

Noronha R. C. R., C. Y. Nagamachi, P. C. M. O'brien, M. A. Ferguson-Smith, y J. C. Pieczarka. 2009. Neo-XY body: an analysis of XY1Y2 meiotic behavior in Carollia (Chiroptera, Phyllostomidae) by chromosome painting. Cytogenetic and Genome Research 124:37-43.

Parish, D. A. 2003. Genome organization, mobile DNA, and Chromosomal evolution in mammals. Tésis de Doctorado. Texas Tech University. Lubbock, EE.UU.

Patton, J., y A. Gardner. 1971. Parallel evolution of multiple sex-chromosomes systems in the Phyllostomatidae bats, Carollia and Choeroniscus. Experientia 27:105-106.

Pieczarka, J. C., C. Y. Nagamachi , P. C. O’brien , F. Yang , W. Rens, R. M. Barros, R. C. Noronha, J. Rissino, E. H. De Oliveira, y M. A. Ferguson-Smith. 2005. Reciprocal chromosome painting between two South American bats: Carollia brevicauda (Schinz, 1821) and Phyllostomus hastatus (Phyllostomidae, Chiroptera). Chromosome Research 13:339-347.

Pine, R. H. 1972. The bats of the genus Carollia. Texas A \& M University, Technical Monograph 8:1-25.

Ramírez-Chaves, H. E., y E. A. Noguera-Urbano. 2010. Lista preliminar de los mamíferos (Mammalia: Theria) del departamento de Nariño - Colombia. Biota Colombiana 11:117-140.

Santos, N., y M. J. De Souza. 1998. Characterization of the constitutive heterochromatin of Carollia perspicillata (Phyllostomidae, Chiroptera) using the base-specific fluorochromes, CMA3 (GC) and DAPI (AT). Caryologia 51:51-60. 
Soları, S., Y R. J. Baker. 2006. Mitochondrial DNA sequence, karyotypic, and Morphological variation in the Carollia castanea species complex (Chiroptera: Phyllostomidae) with description of the new species. Occasional Papers Museum of Texas Tech University 254:1-16.

Solari, S., Y. Muñoz-Saba, J. V. Rodríguez-Mahecha, T. R. Defler, H. E. Ramírez-Chaves, Y F. Trujillo. 2012. Riqueza, endemismo y conservación de los mamíferos de Colombia. Mastozoología Neotropical 20:301-365.

Sтоск, A. D. 1975. Chromosome banding pattern homology and its phylogenetic implications in the bat genera Carollia and Choeroniscus. Cytogenetics and Cell Genetics 14:34-41.

York, H., y M. PAPES. 2007. Limiting similarity in the short-tailed fruit bats. Journal of Zoology 273:1-8.

Sometido: 19 de julio de 2013

Revisado: 23 de julio de 2014

Aceptado: 29 de julio de 2014

Editor asociado: Jesús Maldonado

Diseño gráfico editorial: Gerardo Hernández 
